EPJ Web of Conferences 53, 06007 (2013)

DOI: $10.1051 /$ epjconf/20135306007

(C) Owned by the authors, published by EDP Sciences, 2013

\title{
The need for hard spectra sources of nearby heavy cosmic rays
}

\author{
Andrew M. Taylor
}

ISDC, University of Geneva, Chemin d'Ecogia 16, 1290 Versoix, Switzerland

\begin{abstract}
Using recent Auger energy spectrum and composition analysis results, an investigation is carried out into the requirements placed on the UHECR sources. The the spatial distribution of these sources is investigated along with the energy distribution of UHECR they output. These investigations reveal the need for local UHECR sources which output a hard spectrum of intermediate/heavy UHECR. These results demand that local $(<80 \mathrm{Mpc})$ UHECR sources exist, placing exciting and difficult requirements on the local extragalactic candidate sources. None negligible $(>0.01 \mathrm{nG})$ extragalactic magnetic fields are noted to further strengthen these results.
\end{abstract}

\section{INTRODUCTION}

UHECR, above $10^{19} \mathrm{eV}$ in energy arriving to Earth have been measured for the past several decades. However, only in relatively recent times has the question as to their composition started to become accessible to scrutiny with the sudden increase in statistics due to recent detector developments.

Such developments in the field have only been possible through the construction of massive hybrid ground arrays and fluorescence detectors such as the Pierre Auger Observatory (PAO) in Argentina. With several thousand square-km of collection area, such experiments are able to raise the dismal rates of less than $1 \mathrm{~km}^{-2} \mathrm{yr}^{-1}$ up to a usable level for all but the highest energy flux.

Since the spectral shape alone allows a degenerate set of nuclear species fits, the provision of further composition dependent information is of crucial importance. With such information, deeper studies into the nature and origin of the UHECR can become possible. To this end, the PAO experiment has now released their first measurements of both $\left\langle X_{\max }\right\rangle$ and $\operatorname{RMS}\left(X_{\max }\right)[1,2]$.

As shown in Fig. 1, both the $\left\langle X_{\max }\right\rangle$ and $\operatorname{RMS}\left(X_{\max }\right)$ measurements by the PAO indicate that the composition starts to become heavier at high energies above $10^{18.5} \mathrm{eV}$. On these plots, we also show different colour bands for the theoretical predictions of the expected $\left\langle X_{\max }\right\rangle$ and $\operatorname{RMS}\left(X_{\max }\right)$ values for different (single composition) nuclear species. The width of these bands are provided to convey the present uncertainty which exists in the hadronic models used to calculate both the $\left\langle X_{\max }\right\rangle$ and $\operatorname{RMS}\left(X_{\max }\right)$ values. Interestingly, the use of the spread in this way has recently been bolstered by studies following the first LHC results [3]. Furthermore, as is also seen from the relative widths of these lines, of the $\left\langle X_{\max }\right\rangle$ and $\operatorname{RMS}\left(X_{\max }\right)$ model measurements, the relative uncertainty associated with the $\operatorname{RMS}\left(X_{\max }\right)$ for different species is smaller than that for $\left\langle X_{\max }\right\rangle$.

Curiously, recent corresponding measurements by one of the other leading experiments in the field, the Telescope Array, do not appear to agree with the Auger results [4]. Though an understanding of this conflict is crucial for future progress in the field, we here chose to adopt only the Auger results in order to investigate the consequences that their results naturally imply.

This is an Open Access article distributed under the terms of the Creative Commons Attribution License 2.0, which permits unrestricted use, distribution, and reproduction in any medium, provided the original work is properly cited. 

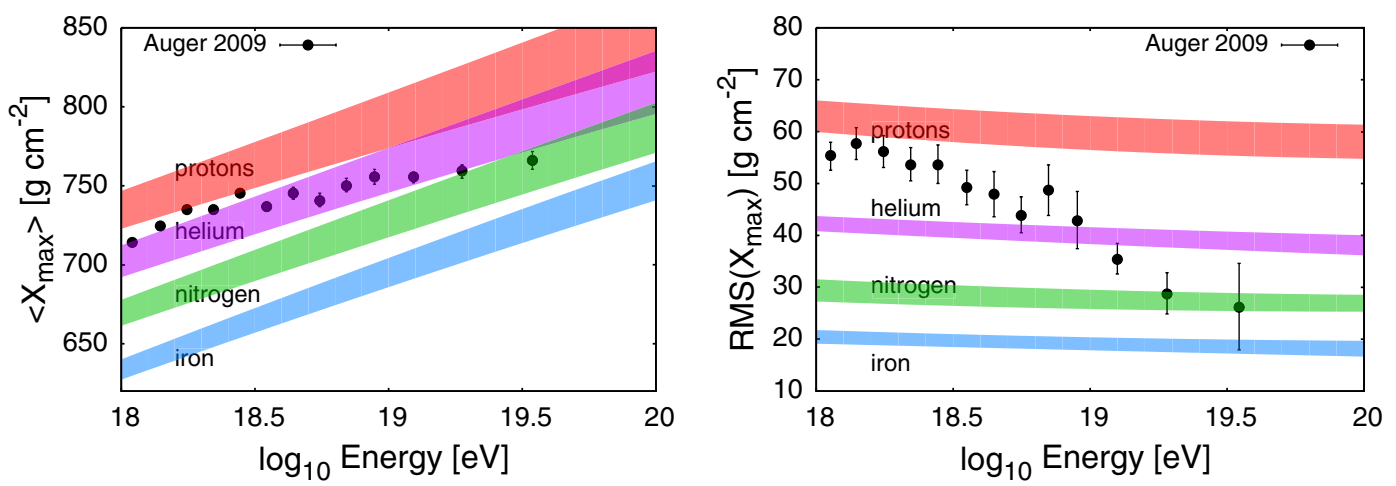

Figure 1. Plots showing PAO measurements of the composition sensitive quantities $\left\langle X_{\max }\right\rangle$ and $\operatorname{RMS}\left(X_{\max }\right)$.

\section{PROPAGATION PHYSICS AND DESCRIPTION}

Before their arrival, UHECRs must propagate across astronomical distances between their source and Earth. The arriving flux from an ensemble of their sources is naturally expected to contain a suppression feature just below the highest energies currently observed. Such a feature results as a consequence of UHECR interactions with background photons. For the case of a proton dominated UHECR composition, photo-pion production interactions will rapidly start to dominate energy losses at energies just below $10^{20} \mathrm{eV}$, leading to a suppression feature being expected at these energies, referred to as the Greisen-Zatsepin-Kuz'min (GZK) cutoff [5, 6].

The expectation of a high energy suppression feature, however, is not unique to the proton composition scenario. Indeed a similar feature is also naturally expected for the case when a significant fraction of the population consists of nuclei. However, for such a scenario, it is instead photodisintegration interactions which lead to the suppression in flux at the highest energies. Subsequently, the identification of a suppression feature at the highest energies is unable to provide much clue as to the underlying source composition.

In order to get a more developed understanding to allow the composition measurement results to be interpreted, the energy loss lengths for different species of UHECR nuclei must be calculated. The calculation of these loss rates follow the general formula,

$$
R_{A, \gamma}=\frac{1}{2 \Gamma_{A}^{2}} \int_{0}^{\infty} \frac{1}{\epsilon_{\gamma}^{2}} \frac{d n_{\gamma}}{d \epsilon_{\gamma}} d \epsilon_{\gamma} \int_{0}^{2 \Gamma_{A} \epsilon_{\gamma}} \epsilon_{\gamma}^{\prime} \sigma_{A \gamma}\left(\epsilon_{\gamma}^{\prime}\right) K_{A \gamma} d \epsilon_{\gamma}^{\prime}
$$

where $d n_{\gamma} / d \epsilon_{\gamma}$ describes the spectral shape of the target photons being interacted with, $\sigma_{A \gamma}$ dictates the interaction rate of the UHECR with these photons, and $K_{A \gamma}$ is the fractional energy loss of the UHECR as a result of the interaction. Example energy loss rate curves for different species are given in [7].

Using a set of such loss/interaction rate curves for the ensemble of nuclear species, the transmutation of species as they propagate through extragalactic radiation fields can be calculated. A Monte Carlo implementation for calculating the temporal evolution of the nuclear state populations was employed in [7]. For these calculations, all possible decay routes through a whole network of possible alternative nuclear isotope states was considered.

With one main decay route dominating the decay network, however, a simplified description employing only a 1D decay chain can be developed. Such a description employs average decay rates between the dominant states in the full decay network which lie along the $1 \mathrm{D}$ chain. Furthermore, in this simplified description, single nucleon losses dominate the decay chain [8], allowing the nuclear state evolution to be described by a simple analytic description [9]. 

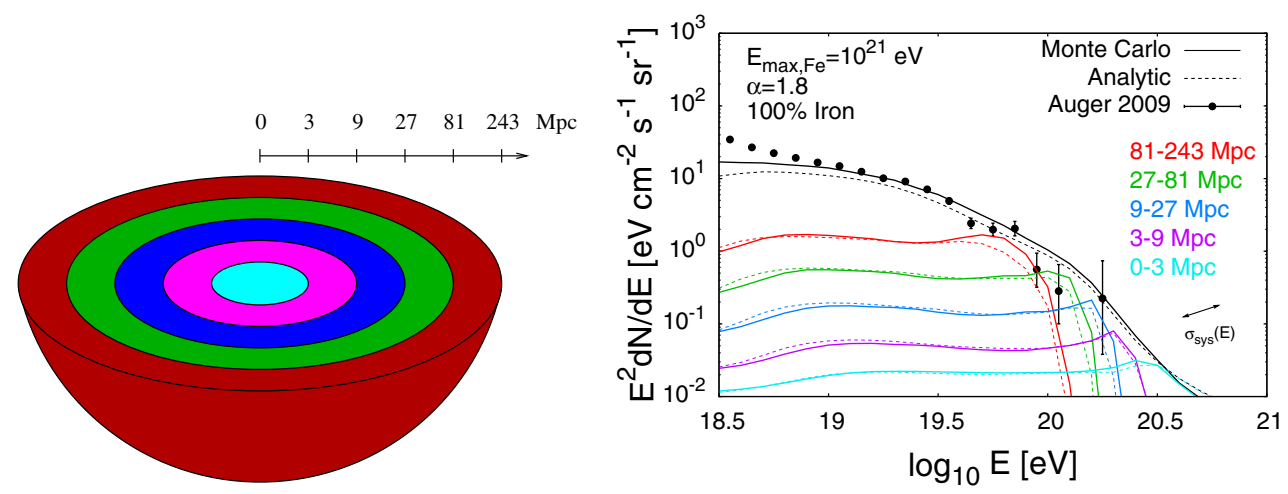

Figure 2. Left-panel: a depiction of the source shells considered. Right panel: a spectral plot showing PAO measurements, Monte Carlo and analytic derived fluxes arriving from the different source shells.

The functional form of the analytic expression describing the spatial distribution of species $A$ at distance $L$ from the source is

$$
\frac{N_{A}\left(E_{A}, L\right)}{N_{A_{\text {ini }}}(E, 0)}=\sum_{m=A}^{A_{\text {ini }}} l_{0} l_{m}^{A_{\text {ini }}-1} e^{-\frac{L}{l_{m}}} \prod_{p=0(\neq m)}^{A_{\text {ini }}} \frac{1}{l_{m}-l_{p}}
$$

where $l_{A}$ is the loss length of species $A$. The functional shape of the analytic expression may be brought into a more recognisable form through the addition of the assumption that the loss lengths for the different species are comparable (valid at least for neighbouring species). Under this assumption, eqn (2) may be re-written as

$$
\frac{N_{A}\left(E_{A}, L\right)}{N_{A_{\text {ini }}}(E, 0)} \approx \frac{\left(L / l_{A}\right)^{A_{\text {ini }}-A}}{\left(A_{\text {ini }}-A\right) !} e^{-\frac{L}{l_{A}}},
$$

whose functional form is the familiar Gaisser-Hillas type function [10], consisting of a power-law rise and an exponential decay. Such an analytic description, built around the dominant nucleon loss chain, allows secondary effects such multi-nucleon losses and pair losses to be taken into account as perturbations to the main solution [11].

With a description of the evolution of the nuclear state populations, the arriving flux and composition from a distribution of sources may be calculated. A comparison of such calculated fluxes for the case of homogeneously distributed sources, each producing UHECR with spectral index $\alpha$ up to a maximum, rigidity dependent cutoff $E_{\mathrm{Fe}, \max }$, for both the analytic description and that of the Monte Carlo, is shown in Figs. 2 and 3. These example comparison plots demonstrate the ability of the analytic description to provide an accurate description of both the arriving spectral shape (Fig. 2) and its composition (Fig. 3). The right-panel of Fig. 2 also shows that the breakdown of the analytic and Monte Carlo arriving fluxes from a homogeneous distribution of sources into different source shell bands are in good agreement out to source distances beyond $243 \mathrm{Mpc}$.

\section{RESULTS}

In order to keep the situation of the UHECR propagation as simple as possible, the presence of extragalactic magnetic fields on UHECR propagation are firstly neglected. Furthermore, with no prior knowledge about the UHECR source population, a "universal" (homogeneous and isotropic) distribution is generally assumed. We adopt this assumption here as a means of investigating signatures of a departure from it. In order to quantify the effect of a different source distribution in this paper, we separate out 

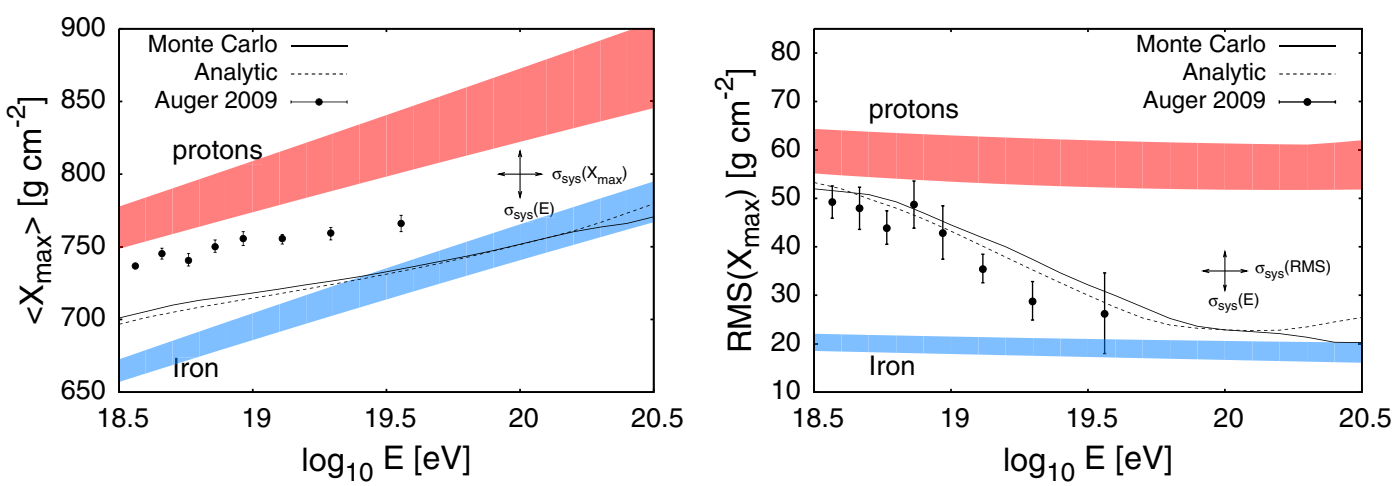

Figure 3. Composition dependence $\left\langle X_{\max }\right\rangle$ and $\operatorname{RMS}\left(X_{\max }\right)$ plots comparing analytic and Monte Carlo based results. For these results a spectral index $\alpha=1.8$ and a maximum source energy of $E_{\mathrm{Fe}, \max }=10^{21} \mathrm{eV}$ have been used.
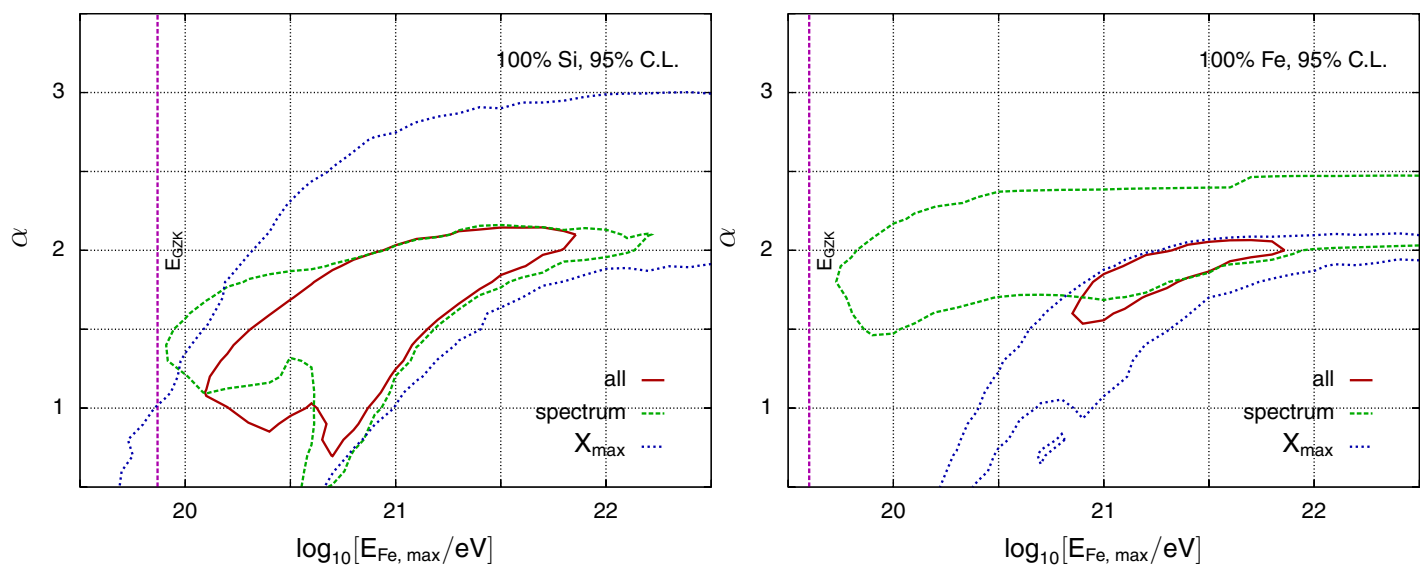

Figure 4. The $95 \%$ C.L. contour plots for silicon and iron only source compositions. These contours indicate the regions of spectral index/maximum energy parameter space where good-fits can be found to the PAO spectral and composition results.

the fluxes produced from source regions with shells of radii 0-3 Mpc, 3-9 Mpc, 9-27 Mpc, 27-81 Mpc, and 81-243 Mpc surrounding the Earth, as depicted in the left-panel of Fig. 2. In this way, the results obtained may be used to encapsulate the effects introduced by a non-"universal" local void of UHECR sources.

As was found in [12], the contour plots in Fig. 4 reveal that sources emitting intermediate-heavy compositions $(A>20)$ UHECR with hard spectral indices $(\alpha \lesssim 2)$ and intermediate energy cutoffs $\left(E_{\mathrm{Fe}, \max } \sim 10^{21} \mathrm{eV}\right)$ are best able to describe the current data. It should also be noted that for light-nuclei type sources the contour space was found to be considerably diminished, with no good-fit contours existing for a proton-only scenario, even at the $99 \%$ C.L.

As shown in Fig. 5, the introduction of a minimum distance to the first source can alter the goodfit contour plots significantly. In particular, for the shell sizes considered, it is seen that for both silicon and iron sources, the $99 \%$ C.L. contours undergo a rapid decrease in size for minimum source distances in the range 9-27 Mpc and 27-81 Mpc respectively. Furthermore, these plots also indicate that for minimum source distances beyond these constrained ranges, uncomfortably large cutoff energies, $E_{\mathrm{Fe}, \max }$, are required by the source population. This result encapsulates the main finding from our study, 
UHECR 2012
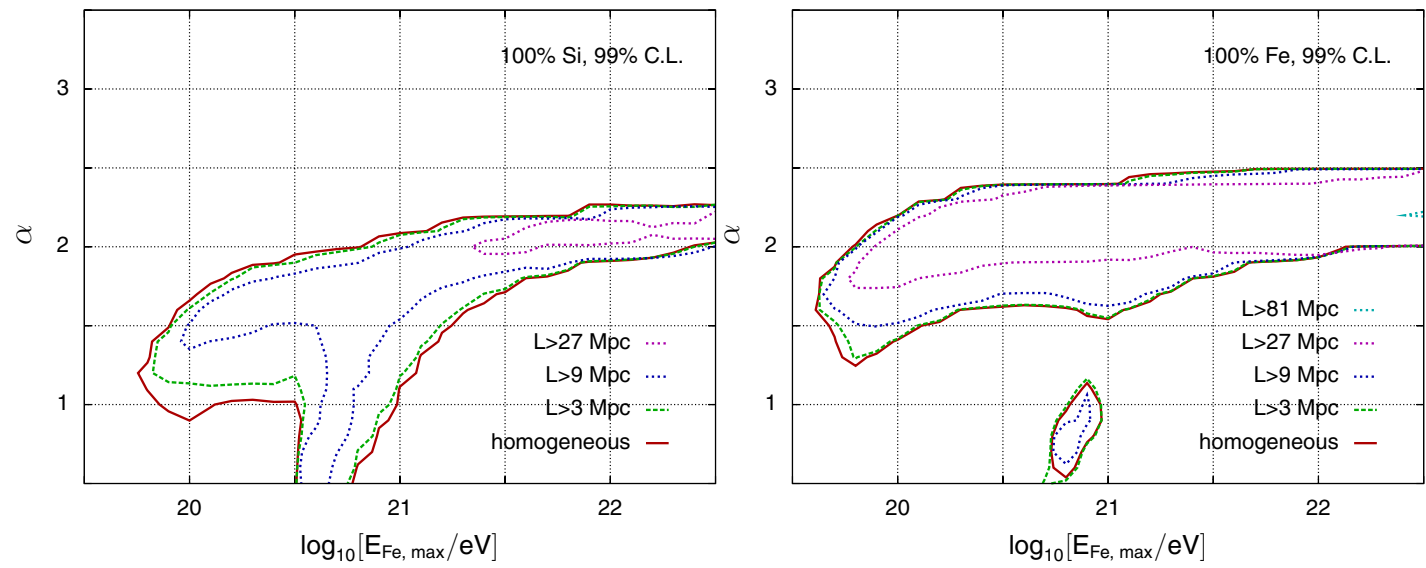

Figure 5. The effect on the $99 \%$ C.L. silicon and iron contour plots by the introduction of a minimum source distance.
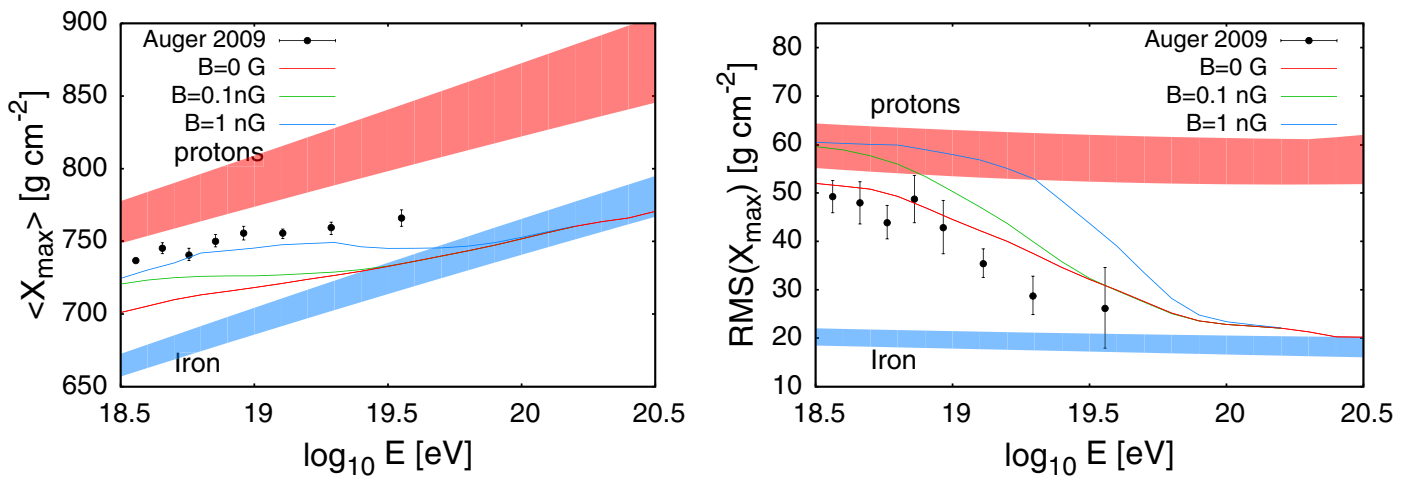

Figure 6. The effect introduced on the arriving spectrum, $\left\langle X_{\max }\right\rangle$ and $\operatorname{RMS}\left(X_{\max }\right)$ by the presence of non-negligible extragalactic magnetic fields and non-zero minimum source distance scale $(L>27 \mathrm{Mpc})$. For these results a spectral index $\alpha=1.8$ and a maximum source energy of $E_{\mathrm{Fe}, \max }=10^{21} \mathrm{eV}$ have been used.

\section{that sources of hard spectra UHECR nuclei sources with local distances, $\sim 40-80 \mathrm{Mpc}$ must exist in local extragalactic space.}

Introducing now the effects of extragalactic magnetic fields, the components of the arriving flux from the different source shells is altered. Consequently, if one (or several) of the nearest source shells depicted in the left-panel of Fig. 2 are void of sources, both the arriving spectrum and composition can be different for different magnetic field strength values. We assume here an extragalactic magnetic field coherence length of $1 \mathrm{Mpc}$, with a power law distribution of magnetic turbulence of the form $P(k) \propto k^{-q}$ (where $k=2 \pi / \lambda$ ), for which $q=5 / 3$ corresponds to a Kolmogorov-type spectrum, $q=$ $3 / 2$ corresponds to a Kraichnan-type spectrum. The effects introduced by the presence of $0.1 \mathrm{nG}$ and $1 \mathrm{nG}$ fields, containing a Kolmogorov type spectrum of turbulence, on previous results are briefly considered.

The effect introduced by the presence of non-negligible extragalactic magnetic fields is shown in Fig. 6. As expected, the presence of non-negligible magnetic fields coupled with a significant distance to the nearest extragalactic source (here taken to be $27 \mathrm{Mpc}$ ) leads to "magnetic horizon" effects [13]. For this scenario, noticeable increase in the amount of photo-disintegration incurred by the UHECR nuclei before arriving at Earth was found. In this way, the presence of non-negligible extragalactic fields 
is seen to strengthen the case for a need of nearby sources, as suggested for the extreme case of $>\mathrm{nG}$ extragalactic fields in [14].

\section{CONCLUSION}

We have here briefly described an investigation into the propagation of UHECR nuclei for intermediate/heavy type composition models. These studies were firstly initiated under the premise that a homogeneous distribution of sources exists. Perturbations on such a homogeneous distribution for the case of a local void of sources were then subsequently investigated in order to determine the furthest distance the nearest sources could be away from Earth and still not violate recent PAO observations. A more detailed description of this work is given in [15].

Both a Monte Carlo and analytic description developed in [9] and [11] were used to describe the propagation and transmutation of the nuclear species in the extragalactic radiation fields. The Monte Carlo and analytic descriptions were demonstrated to agree extremely well for source distance out to and beyond the maximal distance scale considered in this work $(243 \mathrm{Mpc})$.

Taking advantage of the simplicity and speed of the analytic description, a scan over several source parameters describing the injection spectrum output by the set of sources was carried out. Good-fit contours were obtained for the source spectral index, $\alpha$, and the source's maximum rigidity-dependent energy, $E_{\mathrm{Fe}, \max }$. From the results obtained, the need for an intermediate/heavy type source composition with relatively hard injection spectra $(\alpha \lesssim 2)$ was revealed. Furthermore, the introduction of a local void distance out to the nearest-by source was shown to severely deplete the good-fit parameter space. This sudden shrinkage of the good-fit contour size occurred when the void distance scale went beyond $27 \mathrm{Mpc}$ and $81 \mathrm{Mpc}$ for Silicon and Iron type source compositions respectively.

Finally, the introduction of extra-galactic magnetic fields for the case of non-negligible minimum distance to the first source $(27 \mathrm{Mpc})$ was demonstrated to have a potentially noticeable effect on the $\left\langle X_{\max }\right\rangle$ and $\operatorname{RMS}\left(X_{\max }\right)$ fits. However, such $(<\mathrm{nG}$ strength) fields were unable to alter significantly the upper bound on the nearest source distance.

\section{References}

[1] M. Unger, f. t. P. A. Collaboration, arXiv:1103.5857 [astro-ph.HE]

[2] J. Abraham et al. [Pierre Auger Collaboration], Phys. Rev. Lett. 104, 091101 (2010)

[3] D. d'Enterria, R. Engel, T. Pierog, S. Ostapchenko and K. Werner, Astropart. Phys. 35 (2011) 98 [arXiv:1101.5596 [astro-ph.HE]]

[4] J. N. Matthews [Telescope Array Collaboration], Nucl. Phys. Proc. Suppl. 212-213, 79-86 (2011).

[5] K. Greisen, Phys. Rev. Lett. 16, 748 (1966)

[6] G. T. Zatsepin and V. A. Kuz'min, JETP Lett. 4, 78 (1966)

[7] D. Hooper, S. Sarkar and A. M. Taylor, Astropart. Phys. 27199 (2007) [astro-ph/0608085]

[8] J. L. Puget, F. W. Stecker and J. H. Bredekamp, Astrophys. J. 205638 (1976)

[9] D. Hooper, S. Sarkar and A. M. Taylor, Phys. Rev. D 77103007 (2008) [arXiv:0802.1538 [astro$\mathrm{ph}]]$

[10] T. K. Gaisser and A. M. Hillas, ICRC 8353 G (1977)

[11] M. Ahlers and A. M. Taylor, Phys. Rev. D 82123005 (2010) [arXiv:1010.3019 [astro-ph.HE]]

[12] D. Hooper and A. M. Taylor, Astropart. Phys. 33 (2010) 151 [arXiv:0910.1842 [astro-ph.HE]]

[13] M. Lemoine, Phys. Rev. D 71083007 (2005)

[14] T. Piran, arXiv:1005.3311 [astro-ph.HE]

[15] A. M. Taylor, M. Ahlers and F. A. Aharonian, Phys. Rev. D 84, 105007 (2011) [arXiv:1107.2055 [astro-ph.HE]] 